\title{
Simultaneous Correction for Interscan Patient Motion and Geometric Distortions in Echoplanar Imaging
}

\author{
T. Ernst, ${ }^{*}$ O. Speck, L. Itti, and L. Chang
}

\begin{abstract}
A method is presented for simultaneous correction of linear geometric distortions and interscan patient motion in echoplanar imaging (EPI). The technique does not require the acquisition of specialized scans other than high-resolution magnetic resonance images. The method is based on a generalized surface-based coregistration algorithm, which accounts for a complete 3-dimensional affine transformation, i.e., rotations, translations, scaling, and shearing, between two volumetric image data sets. Any minimally distorted high-resolution scan may serve as a reference data set, to which the EPI data set is matched. The algorithmic accuracy was assessed using simulated data sets with known affine distortions. The deviation of the parameters determined by the coregistration program from the true values typically was $1 \%$ or less. Precise alignment of functional and anatomic information will be important for many future clinical applications. Magn Reson Med 42:201-205, 1999. (c) 1999 Wiley-Liss, Inc.
\end{abstract}

Key words: MRI; geometric distortions; patient motion; coregistration; echoplanar imaging

Several advanced "functional" MRI modalities, such as perfusion, diffusion, and functional MRI, are based on ul trafast scanning techniques, most commonly echoplanar imaging (EPI). Since these functional scans usually have relatively low resolution (typically $64 \times 64$ ) and contrast, and thus show poor anatomic detail, it is advantageous to map the functional information onto high-resol ution structural scans. Such mapping may be crucial for certain clinical applications, for instance, to identify the precise anatomic location of activated areas in functional MRI.

However, the exact spatial relationship between functional and anatomi c scans may be impaired by two factors: 1) interscan patient motion, which may cause a translation and rotation between the two sets of scans and which may occur even within the same session; and 2) geometric distortions of echoplanar images. Geometric distortions in EPI are a result of deviations of the k-space trajectory from its ideal path dueto inhomogeneities of the static magnetic field. Unlike standard spin warp imaging techniques, EPI is very sensitive to even rel atively small fiel d inhomogeneities, due to the nature and the long duration of the EPI trajectory. These distortions are most pronounced in the phase direction since this direction is acquired with a low effective bandwidth in EPI $(1,2)$. A linear magnetic field gradient in the phase-encoding direction will cause a stretching or shrinking (i.e., scaling) of the reconstructed

Harbor UCLA Research and Education Institute, Torrance, California

Grant sponsor: NIH (Scientist Development Award for Clinicians); Grant number: DA 00280; Grant sponsor: State of California Universitywide AIDS Research Program; Grant number: CC97 LA 175.

${ }^{*}$ Correspondence to: Thomas Ernst, Department of Radiology, Harbor UCLA Medical Center, 1124 W. Carson Street, N-11, Torrance, CA 90502.

Received 4 November 1998; revised 31 January 1999; accepted 2 March 1999

() 1999 Wiley-Liss, Inc. echoplanar image in the phase direction, whereas a linear magnetic field gradient perpendicular to the phaseencoding direction will cause a shearing of the reconstructed image in the phase di rection $(1,2)$.

This paper describes a computer al gorithm that simultaneousl y corrects for patient motion and first-order geometric distortions (scaling and shearing) in EPI-based "functional" MRI modalities. Unlikeother techniques that correct for geometric distortions in EPI (1-6), our method does not rely on the acquisition of additional, special scans (for instance, to obtain field maps) other than a regular highresol ution MRI. A general ized coregistration al gorithm that accounts for a complete 3-dimensional (3D) affine transformation, i.e., rotations, translations, scaling, and shearing, is applied to two vol umetric image data sets. A minimally distorted high-resolution scan serves as a reference data set, to which the EPI data set is matched. In a first step, the true geometry (surface) of the object of interest is extracted automatically from the high-resolution reference scan. Next, the geometry of the displaced and distorted EPI scan is extracted automatically. In a second, iterative step, the geometry of the EPI scan is subjected to affine transformations until it best matches that of the reference scan. More specifically, we scale, shear, rotate, and shift the brain surface from the EPI scan to match the brain surface from the corresponding reference scan. The starting point for the matching is the relative position of the two brain scans known from the scanner. In the final processing step, the original echoplanar images are resliced according to the affine transformation; the resliced images are then in register with the high-resolution scan. The algorithm can readily be modified to perform only a subset of the affine transformations, such as shearing and scaling only, to correct for geometric distortions.

\section{MATERIALS AND METHODS}

All data were acquired on a 1.5 T whole-body scanner (GE Signa 5.7, Milwaukee, WI) equipped with fast shielded gradients (SR 120). The high-resolution reference scan was obtai ned with a fast spin-echo inversion recovery sequence (inversion time $120 \mathrm{msec}$, echo time $32 \mathrm{msec}$, repetition time $4000 \mathrm{msec}, 3.5 \mathrm{~mm}$ contiguous slice, matrix $256 \times$ 256). Gradient-recalled blipped EPI scans were acquired with echo times of $30 \mathrm{msec}$ (perfusion MRI) or $60 \mathrm{msec}$ (functional MRI) and a repetiti on time of $2500 \mathrm{msec}(6 \mathrm{~mm}$ slice thickness, 2 mm gap, 16-20 slices). In addition, EPI scans with intentionally misadjusted shim values were acquired in a healthy volunteer. For the EPI scans, the linear shims were optimized using the automatic shim procedure available on the scanner. We assume a coordinate system where $x$ characterizes the left-right direction, $y$ 
corresponds to the anterior-posterior direction, and $z$ is along the long body axis (inferior-superior).

Theal gorithm to determinetheoptimal affinetransformation was implemented as an expansion of a customized coregistration software package developed in our laboratory $(7,8)$. Since the original coregistration program used only translations, rotations, and scaling to match two different scans, we modified the program to include shearing for a full 3D affine transformation. The coregistration program is based on a surface matching al gorithm (7) and involves two primary steps. In the first primary step, the brain surfaces from the two scans (high-resolution reference and EPI) are extracted semi-automatically using an extension of the techniques described by Alpert et al. (9) and Mangin et al. (10). First, pixels with a signal intensity outside that of the brain intensity range are excluded by applying an intensity window that is automatical ly calculated from the intensity histogram of the entire scan. The resulting binary $3 \mathrm{D}$ volume within the accepted intensity range is then subjected to isotropic 3D binary morphological erosion to separate the brain from surrounding tissues; fast isotropic 3D erosion is obtained by thresholding an anisotropic chamfer distance map $(7,11)$. Next, the eroded binary brain is isolated from any remaining extracerebral structures (tongue, eyes, etc.) using an 8-connected 3D flooding algorithm starting at the centroid of the eroded volume. To recover the brain size from the eroded and extracted binary brain, a 3D binary morphological dilation is applied by thresholding the anisotropic chamfer distance map. Since the dilated brain is smooth and slightly (1-2 mm) larger than the ori gi nal brain, the brain surface is refined by masking the dilated volume with the initial thresholded (non-eroded) binary volume, in which most brain surface contours are already present. Finally, the brain surface contours are converted to a set of $3 D$ vertices.

In the second primary step, the brain surface from the EPI scan is iteratively matched to that from the highresolution scan. The brain surface from the EPI scan is considered mobile, whereas the brain surface from the high-resolution scan is a fixed reference. Therefore, 3D vertices are used to describe the EPI brain surface, and the volume of brain contours is used to describe the highresolution reference surface. The high-resolution brain contours are used to calculate an anisotropic chamfer distance map; the chamfer map contai ns an approximati on to the closest Euclidian distance between a given pixel and the brain surface $(7,12)$. The mobile surface data points, i.e., the EPI brain surface, which are stored as 3D vertices v, are iteratively transformed to new vertices $v$ ' using a full 3D affine transformation with 12 parameters:

$$
\mathrm{v}^{\prime}=\mathrm{A} \mathrm{v}+\mathrm{T},
$$

where the vector $\mathbf{T}$ represents a translation (three parameters), and the $3 \times 3$ matrix A (nine parameters) may be viewed to represent three rotations, three scaling factors, and three shearing factors. Only three of six possible shearing transformations are used in such a representation; the remaining three shearing transformations are linearly dependent on the three scaling, rotation, and shearing transformations. The known offsets (translations) of the two data sets are used to obtain an appropriate starting point for the iterative algorithm. The mismatch between the two brain surfaces is then evaluated by calculating a generalized distance measure $D$ from the chamfer map and the positions of the transformed vertices. The distance measure is an approximation to the mean squared Euclidian distance between the vertices on the mobile surface and the reference surface. Because the EPI scans or the high-resolution scans may not cover the entire brain, the generalized distance measure $D$ is defined in such a way that it is possible to match scans with incomplete overlap (7). Since the resulting distance measure is not a continuous variable, Powell's minimization algorithm for nonregular functions is used to minimize iteratively the distance $D$ between the two brain surfaces (13). After convergence of the surface-matching process, the EPI images are resliced according to the opti mal affine transformation using a 3D trilinear interpolation.

To validate our affine coregistration and to determine its accuracy, simulations on data sets with known deformations were performed (Fig. 1). To obtain realistic simulations, a segmented high-resolution $(256 \times 256)$ data set of a head (Fig. 1, top row) was transformed with various affine transformations, and down-sampled to the lower resolution of an EPI scan $(64 \times 64$; Fig. 1, middle row). The resulting data set was then coregistered with the original high-resolution template using the al gorithms described above, and resliced according to the optimal affine parameters (Fig. 1, bottom row). As the starting point for the coregistration, the two centers-of-mass of the two data sets were aligned. The affine parameters after coregistration and the generalized distance measure $D$ between the two brain surfaces were used to assess the quality of the al gorithm.

\section{RESULTS}

The graphs in Fig. 2 summarize the results of the simulation studies. Scal ing factors between 0.7 and 1.3, applied to the original data set (true values), were recovered by the coregistration al gorithm with an average accuracy of 0.009 (x-direction, in-plane) and 0.011 (z-direction, out of plane) (Fig. 2, top left). Along the x-direction (in-plane), translations of up to $30 \mathrm{~mm}$ were determined with an average accuracy of $0.16 \mathrm{~mm}$ (maximum deviation $<0.4 \mathrm{~mm}$ ), whereas translations along the $z$-axis (out of plane) were reconstructed with an average accuracy of $0.40 \mathrm{~mm}$ (maximum deviation $0.65 \mathrm{~mm}$ ) (Fig. 2, top right). Similarly, shearing factors between \pm 0.3 in the $y$-direction were recovered with an average accuracy of 0.0019 (maximum deviation 0.0035 ), while rotations of up to $30^{\circ}$ about the z-axis were determined with an average accuracy of 0.29 degrees (maximum deviation 0.68 degrees). Essentially independently of the affine transformation used for the simulation, the mean squared distance between the original and final resliced brain surfaces was close to $0.9 \mathrm{~mm}$; the maximum distance found was $1.1 \mathrm{~mm}$.

Figure 3 exemplifies the utility of our technique to correct for geometric distortions in images of the human brain. The extracted brain surface from the high-resolution scan (Fig. 3a; white line) cl osely matches the surface of the 


\section{Slice 7}
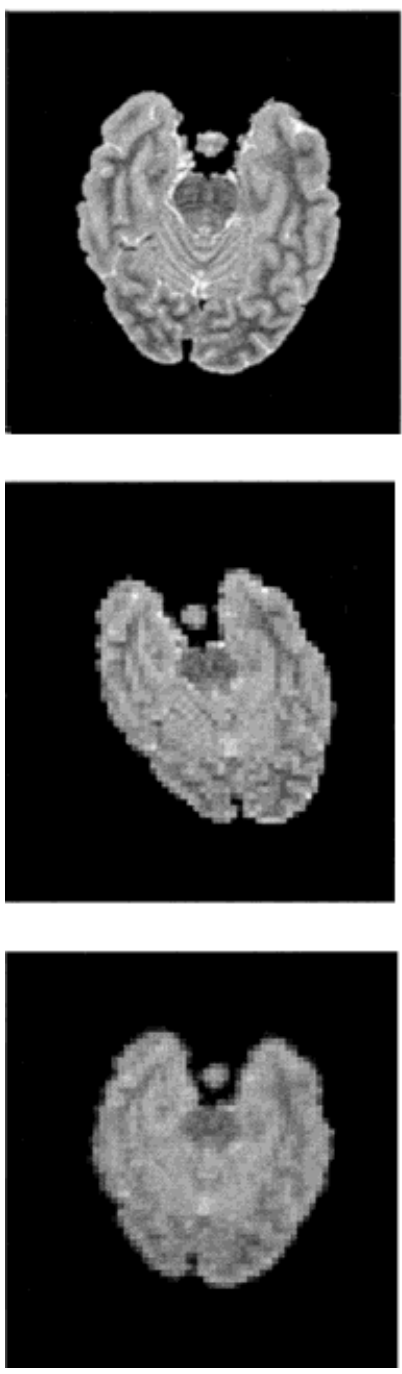

Slice 9
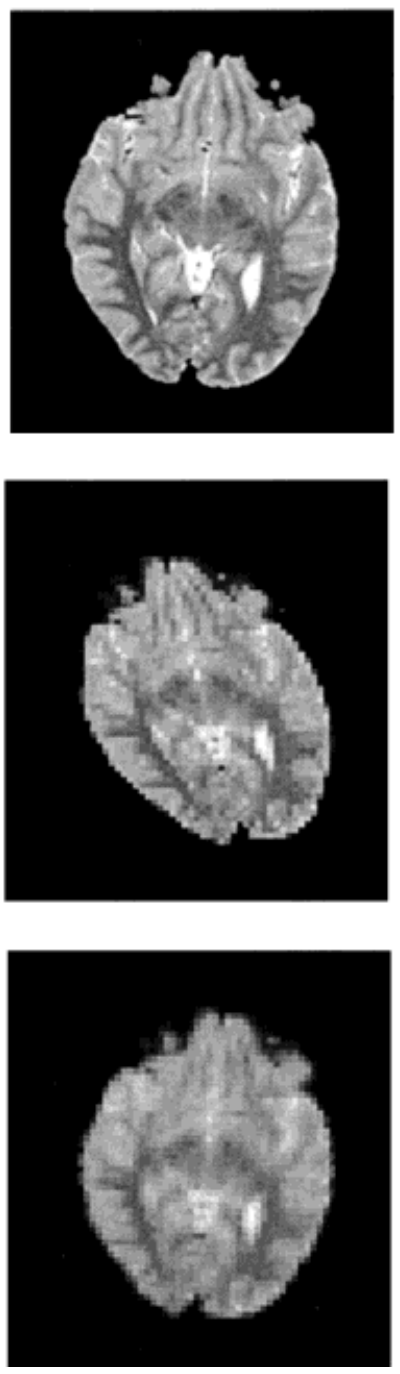

Slice 11
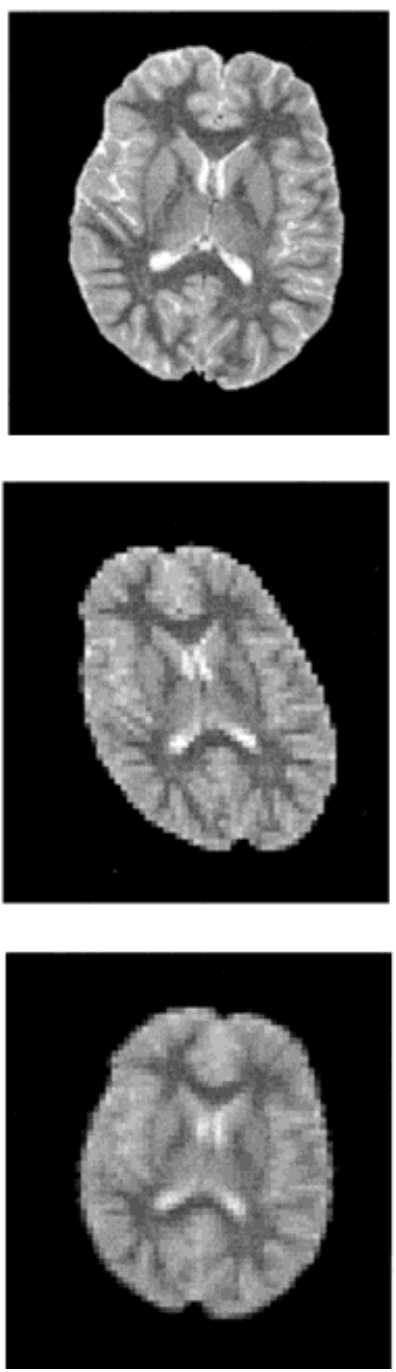

Slice 13
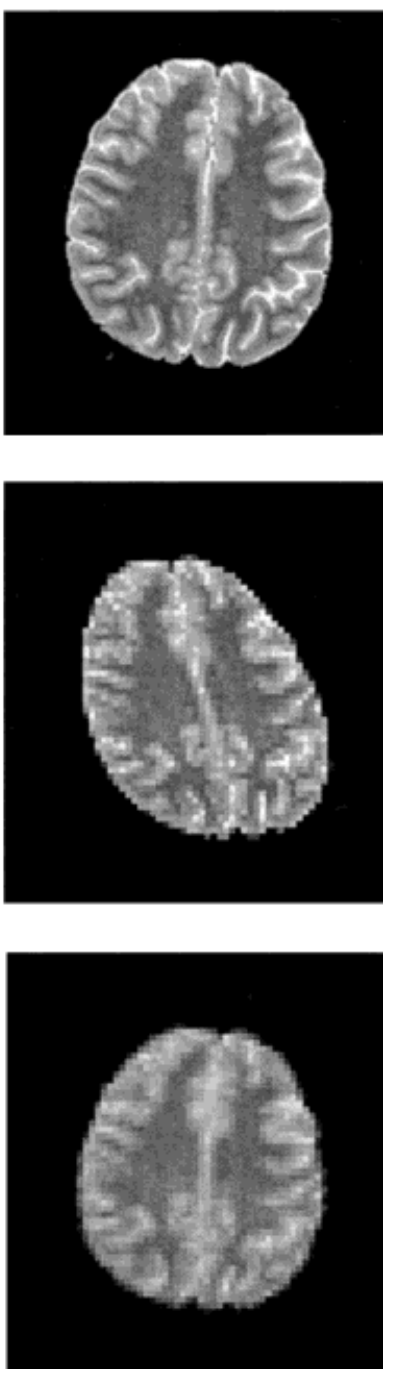

FIG. 1. Examples of MRI images used for the simulation studies. The top row shows four of the original high-resolution slices after brain segmentation (matrix size, $256 \times 256$ ). The middle row (distorted, low-resolution) shows the same images after distorting the original images with an affine transformation ( 0.2 shear in y direction, followed by $20^{\circ}$ rotation about the $z$-axis), and then down-sampling to a matrix size of $64 \times 64$. The bottom row (coregistered, low-resolution) displays the result of coregistering the brain surface from the distorted low-resolution images with the surface from the high-resolution scan, and reslicing according to the matching affine transformation (using trilinear interpolation). The affine parameters found by the coregistration algorithm were a y-shear of 0.1994 and a z-rotation of 20.02 degrees, very close to the initially applied parameters.

echoplanar image with optimal shim setting (Fig. 3b), with a mean squared distance of $3.1 \mathrm{~mm}$. In contrast, the echoplanar image exhi bits substantial geometric distortion (Fig. 3c) when the $x$ - and $y$-shims are misadjusted (mean squared distance, $7.5 \mathrm{~mm}$ ). Our correction al gorithm removes these distortions (Fig. 3d), with a mean squared distance between the original high-resolution and the final corrected brain surface of $4.0 \mathrm{~mm}$.

\section{DISCUSSION}

Our al gorithm combines i mage processing techniques from two different fiel ds, i.e., coregistration and post-processing of echoplanar images, to correct for geometric distortions.
Coregistration is most commonly employed to align data from two different imaging modalities, for instance, MRI data sets with nuclear medicine scans such as positron emission tomography (PET) or single-photon emission tomography (SPECT). However, sincemost EPI-based imaging techniques show relatively poor anatomic details, it may be necessary to register such scans with highresolution scans from the same patient, especially if patient motion is suspected. In our experience, patients who obtai $n$ a perfusion $\mathrm{MRI}$ scan sometimes move slightly after they have been taken out of the magnet for the intravenous catheter placement pri or to the gadolinium bolus injection. When precise alignment of anatomical and functional, i.e., perfusion, information is necessary for clinical purposes, it 


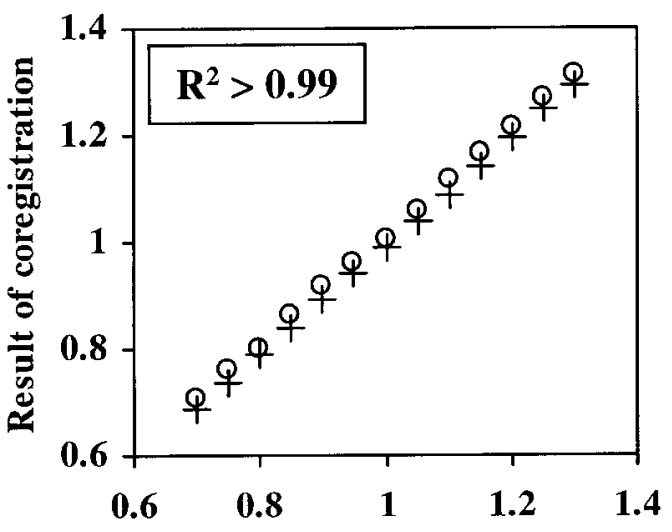

True scaling factor

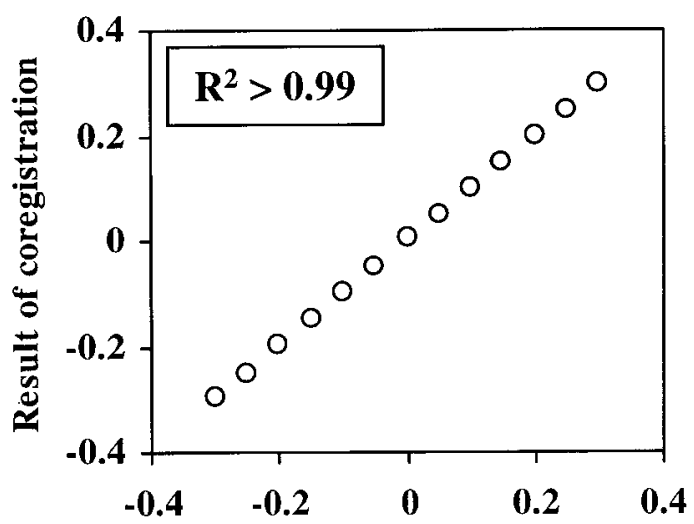

True shearing factor

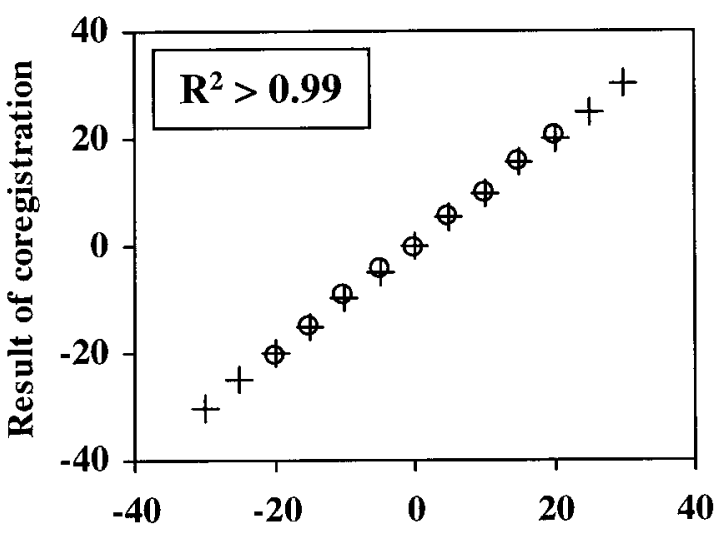

True displacement $(\mathbf{m m})$

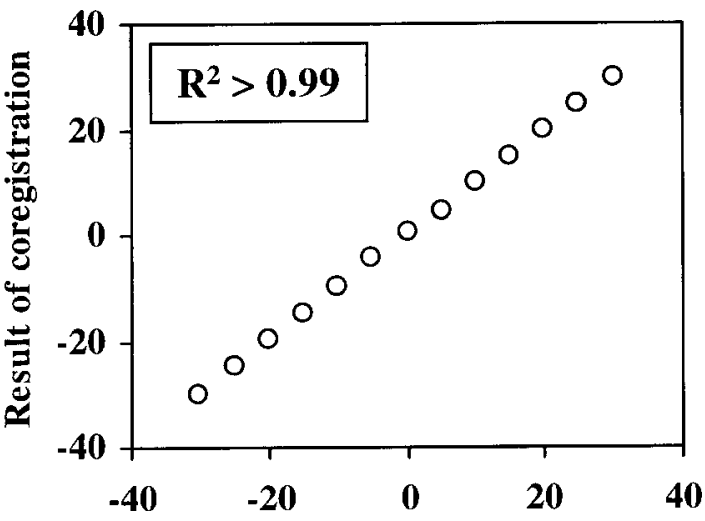

True rotation (degree)

FIG. 2. Results of the simulation studies. Each graph displays the affine parameter found by the coregistration program (y-axis) after applying a certain affine transformation to the original high-resolution MRI scan (true values, $x$-axis). The individual graphs show the result of coregistration after initially applying various degrees of $x$ - (plus marks) and z-scaling (circles; top left), $x$ - (plus marks) and z-translation (circles; top right), $y$-shearing (bottom left), and z-rotation (bottom right). The affine parameters found by the coregistration program are in excellent agreement with the true values.

will be imperative to consider such potential patient motion by co-registering the functional data set with the high-resolution anatomical scans. Our algorithm solves this problem, and simultaneously corrects for first-order geometric distortions in the EPI scans.

Several techniques have been proposed to correct for image distortions in EPI scans. Reese et al. (14) demonstrated that an analytic procedure for shimming the static magnetic field prior to the data acquisition yields good image quality in the human brain. Other groups have described methods to utilize phase, eddy-current, or static magnetic field maps to correct for geometric distortions of echoplanar images (1-6). All these techniques require additional, special scans to extract the information used for the correction. In contrast, our technique can be applied retrospectively even when these special techniques are unavailable or fail due to technical difficulties or patient motion. On the other hand, our technique also has some limitations in that local, high-order field inhomogeneities, such as those found in the temporal lobe or the orbitofrontal regions $(2,15)$, cannot be corrected, and because si gnal losses due to magnetic field inhomogeneities cannot be recovered.

Our computer simulations show that our algorithm works with great accuracy. When simulated data sets with known affine transformation parameters (true val ues) were subjected to our algorithm, the original transformation parameters were typically determined with a precision of $1 \%$. For example, in-plane and out-of-plane translations were recovered with typical accuracies of $0.16 \mathrm{~mm}$ and 0.4 $\mathrm{mm}$, respectively, which is an order of magnitude smaller than the resolution of the down-sampled, transformed images ( $3 \mathrm{~mm}$ in-plane resolution and $8 \mathrm{~mm}$ distance between the centers of adjacent slices). Similarly, rotations of up to $30^{\circ}$ were recovered with an accuracy of typically $0.3^{\circ}$. The mean squared distance between the initial highresolution and the final reconstructed low-resolution surfaces was below $1 \mathrm{~mm}$. In contrast, the typical mean squared distance between high-resolution scans and in vivo EPI scans was $2-3 \mathrm{~mm}$, similar to the in-plane image resolution. Thus, the surface matching error with in vivo scans appears to be dominated by experimental factors 


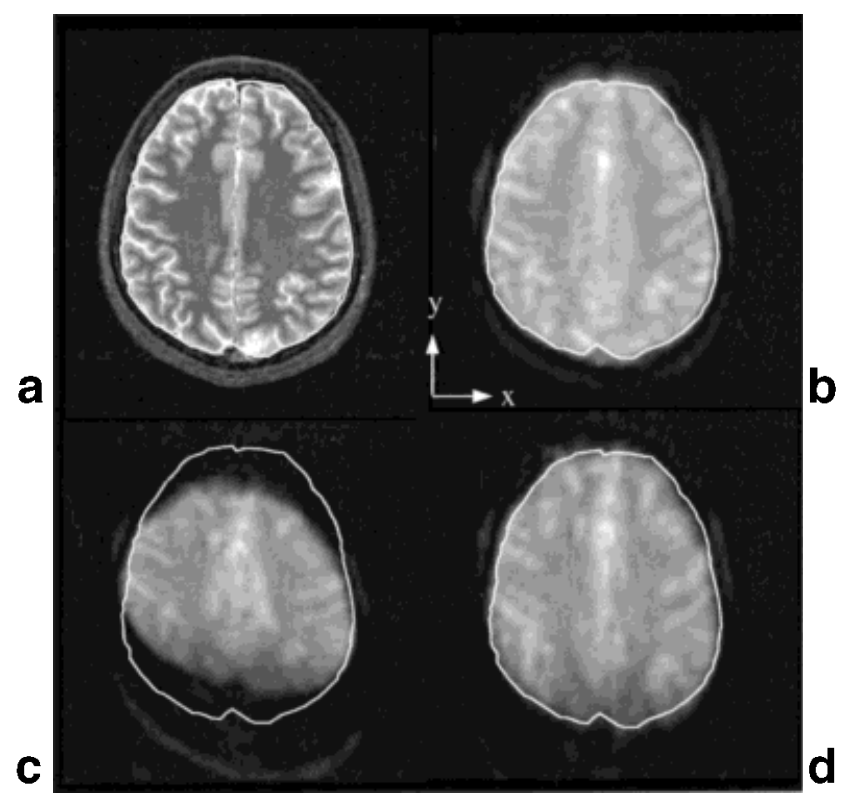

FIG. 3. Axial MRI images of a human brain acquired with a high-resolution T2-weighted sequence (a), and a single-shot EPI sequence with (b) optimal shim setting and (c) intentionally misadjusted $\mathrm{x}$ - and $\mathrm{y}$-shim. The image shown in $\mathbf{d}$ was obtained from image $c$ after correcting for linear geometric distortions. The EPI scans were acquired with read direction along the horizontal $(x)$ and phase direction along the vertical $(y)$ axes. The white line represents the brain contour from the high-resolution scan.

such as the presence of non-linear magnetic field inhomogeneities and inaccuracies in the surface extraction of the EPI scans.

In summary, we present a method for simultaneous correction of linear geometric distortions and patient motion in EPI. Our method does not require the acquisition of any specialized scans other than a high-resolution MRI, and it can be applied retrospectively, si nce high-resolution anatomic scans are usually avail able. Our method is useful for correcting geometric distortions in EPI-based perfusion, diffusion and functional (BOLD) MRI scans if the automatic shim procedure fails, if patients move between the high-resolution scans and the "functional" scans, and for better comparison of repeat "functional" MRI scans performed in separate sessions. Precise alignment of func- tional and anatomic information will be important for many future clinical applications.

\section{ACKNOWLEDGMENTS}

This study was supported in part by the NIH (Scientist Development Award for Clinicians DA 00280, to L.C) and the State of California Universitywide AIDS Research Program to the UCLA Care Center (CC97 LA 175). We also thank the technical staff and acknowledge support from the Harbor-UCLA Imaging Center/InSight, Inc., Torrance, CA.

\section{REFERENCES}

1. Sekihara $\mathrm{K}$, Kohno $\mathrm{H}$. Image restoration from non-uniform static field influence in modified echo-planar imaging. Med Phys 1987:14:10871089.

2. Jezzard P, Balaban, RS. Correction for geometric distortion in echoplanar images from B0 field variations. Magn Reson Med 1995;34:6573.

3. Haselgrove JC, Moore JR. Correction for distortion of echo-planar images used to cal culate the apparent diffusion coefficient. Magn Reson Med 1996;36:960-964.

4. Wan X, Gull berg GT, Parker DL, Zeng GL. Reduction of geometric and intensity distortions in echo-planar imaging using a multireference scan. Magn Reson Med 1997;37:932-942.

5. Jezzard P, Barnett, AS, Pierpaoli C. Characterization of and correction for eddy current artifacts in echo-planar diffusion imaging. Magn Reson Med 1998;39:801-812.

6. Reber PJ, Wong EC, Buxton RB, Frank LR. Correction of off resonancerelated distortion in echo-planar imaging using EPI-based field maps. Magn Reson Med 1998;39:328-330.

7. Itti L, Chang L, Mangin JF, Darcourt J, Ernst T. Robust multimodality registration for brain mapping. Hum Brain Mapping 1997;5:3-17.

8. Itti L, Chang L, Ernst T, Mishkin F. Improved 3-D correction for partial volume effects in brain SPECT. Hum Brain Mapping 1997;5:379-388.

9. Alpert NM, Berdichevsky D, Levin Z, Morris ED, Fishman AJ. Improved methods for image registration. Neuroimage 1996;3:10-18.

10. Mangin JF, Frouin V, Bloch I, Bendriem B, Lopez-Krahe J. Fast nonsupervised $3 D$ registration of PET and MRI images of the brain. J Cereb Blood Flow Metab 1994;14:749-762.

11. Wacken PFM. Chamfer metrics in mathematic morphology. J Math Imaging Vision 1994;4:233-253.

12. Borgefors G. Distance transformations in digital images. Comp Vision Graph Image Proc 1986;34:344-348.

13. Powell MJD. An efficient method for finding the minimum of a function of several variables without calculating derivatives. Comput J $1964 ; 7$ : 155- 163.

14. Reese TG, Davis TL, Weisskoff RM. A utomated shimming at 1.5T using echo-planar image frequency maps. J Magn Reson Imaging 1995;5:739745.

15. Ernst T, Hennig J. Double-volume ${ }^{1} \mathrm{H}$ spectroscopy with interleaved acquisitions using tilted gradients. Magn Reson Med 1991;20:27-35. 